\title{
On Stabilizability of LTI Systems across a Gaussian MAC Channel
}

\author{
Jie Liu ${ }^{1}$ and Vijay Gupta ${ }^{2}$
}

\begin{abstract}
In this paper, the problem of stabilizing two scalar plants across a Gaussian multiple access channel (MAC) is addressed. Both states are encoded separately and sent across a shared MAC channel to a joint decoder/controller. A necessary condition for stabilizing such a system in the mean square sense is obtained using information-theoretic tools. This condition establishes the relationship between the magnitude of the unstable eigenvalues and the capacity region of Gaussian feedback multiple-access channel. A time-division encoding scheme along with a switched linear control law is proposed to achieve a rate region that is sufficient for stabilizability. The problem is studied both under the assumption that the two encoders can coooperate by sharing information and when they cannot.
\end{abstract}

\section{INTRODUCTION}

The stabilizability of networked control systems has attracted much attention recently. Much work has considered systems whose components (plant, controller, sensor and actuator) are connected using point-to-point channels. The stabilizability of such systems is influenced by communication constraints such as packet drops, channel delay, limited data rate [1], and so on. Particularly relevant to this paper is the stream of work that has considered stabilization across additional white Gaussian noise (AWGN) channels. For such channels, a tight data rate bound has been obtained for stabilizability in the mean square sense [2], [3]. This bound relates the eigenvalues of the open loop plant to the minimal signal-to-noise ratio of the AWGN channel that is required for stabilizability. In [4], a necessary condition on the Shannon capacity of the AWGN channel needed to achieve almost surely asymptotically stability was presented. Sufficient conditions for stability over Gaussian product channel [5], Gaussian relay channel [6], Gaussian MAC and broadcast channel [7] have also been presented.

In this paper, we consider the case that when two processes need to be controlled across a shared Gaussian multiple access (MAC) channel. A MAC channel has two encoders, but the channel output is the sum of two channel inputs and an additive white Gaussian noise. While distributed control has been considered for some channel models [8], [9], [10], typically these works assume the communication network to be a collection of point to point channels. There is much less work available for control across MAC channels, in which transmission from one source interferes with the transmission from another source. While one obvious possibility is to perform some scheduling for the two sources, it is known

\footnotetext{
${ }^{1}$ J.Liu is a research assistant in Department of Electrical Engineering, University of Notre Dame, Notre Dame, IN 46556, USA

${ }^{2}$ V.Gupta is with the Department of Electrical Engineering, University of Notre Dame, Notre Dame, IN 46556, USA
}

that such a strategy leads to rate loss [11]. We are aware of only one work that considers control across a MAC channel. In [7], the authors proved that a specific point in the plane defined by the rates at which the two encoders transmit is sufficient for stabilizability.

In this paper, we characterize more fully the rate region for the MAC channel required for stabilizability of the two plants. We consider two cases: one in which the two encoders can cooperate by exchanging information and another in which no information is exchanged between the encoders. We provide necessary and sufficient conditions for stabilizability that provide inner and outer bounds on the rate region required for stabilizability. In particular, for the case with information exchange, the two bounds coincide. On the other hand, if no information is being exchanged, there is still a gap between the two bounds ${ }^{1}$. As was pointed out in [12], the Shannon capacity of a communication channel may not be sufficient to characterize the rate required to stabilize a control system across it. Rather, the notion of anytime capacity, which is in general less than the Shannon capacity, is needed. For control across a point to point AWGN channel, the two capacities are equal and the Shannon capacity characterization of the AWGN channel suffices to provide necessary and sufficient conditions for stabilizability. For the case when the encoders exchange information, our results, thus, indicate that the anytime capacity region of the MAC channel is indeed the same as the Shannon capacity region. For the case without information exchange, we obtain necessary conditions by considering the Shannon capacity region. However, the fact that there is a gap between the necessary and sufficient conditions leads to the conjecture that anytime capacity in this setting may not be the same as the Shannon capacity.

The rest of the paper is organized as follows. Section II formulates the problem and presents the main results. Section III provides the proofs for the case when there is no information exchange between the encoders. Section IV proves the results under the assumption that the encoders exchange information. Section V concludes the paper.

\section{Problem Statement and Main Results}

Consider two scalar plants, with state equations given by

$$
x_{i, t+1}=\lambda_{i} x_{i, t}+u_{i, t} \quad \text { for } \quad i=1,2,
$$

where $x_{i, t} \in \mathbb{R}$ is the state of the $i$-th plant at time $t$ and $u_{i, t} \in \mathbb{R}$ is the corresponding control input. The eigenvalues

\footnotetext{
${ }^{1}$ In this case, the sufficient conditions that we provide include the conditions in [7] as a special case
} 


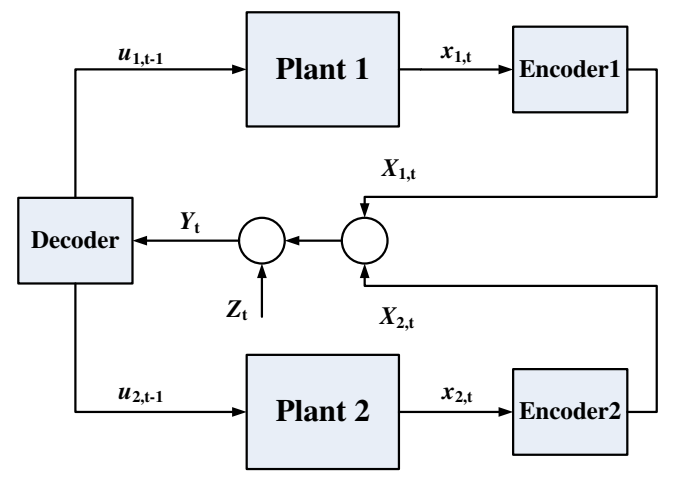

Fig. 1. Two scalar plants with an MAC channel

are assumed to be unstable $\left|\lambda_{i}\right|>1$. We assume that the initial values $x_{i, 0}$ are mutually independent random variables with zero mean and variance $\sigma_{i, 0}^{2}$. As illustrated in Figure 1, the state of each plant is encoded and transmitted across a shared MAC channel. In particular, the sensor $i$ is assumed to observe the exact value of state $x_{i, t}$ at each step $t$. This state value is encoded into the value $X_{i, t}=f_{i}\left(t, x_{i, t}\right)$ and transmitted. We consider an average power constraint of the form $\lim _{T \rightarrow \infty} \frac{1}{T} \sum_{t=1}^{T} \mathbb{E} X_{i, t}^{2} \leq P_{i}$.

The channel output is given by

$$
Y_{t}=X_{1, t}+X_{2, t}+Z_{t},
$$

with zero mean white Gaussian noise $Z_{t} \sim \mathcal{N}(0, N)$ that is independent of the other random variables in the system. This output is observed by the two decoders. Since the decoders are collocated, they can be considered to be effectively a single decoder or controller. The controller computes two control inputs $u_{i, t}=g_{i}\left(t, Y^{t}\right)$ where $Y^{t}$ represent the sequence $\left\{Y_{1}, Y_{2}, \ldots, Y_{t}\right\}$. The control input $u_{i, t}, i=1,2$ is transmitted and applied to plant $i$. For such a system, we define stability as follows.

Definition 1: (Mean Square Stability) The system (1) is stable in the mean square sense if and only if

$$
\lim \sup _{t \rightarrow \infty} \mathbb{E}\left[x_{i, t}^{2}\right]=0, \quad i=1,2 .
$$

If there exist design parameters $f_{i}(\cdot), g_{i}(\cdot)$, for $i=1,2$ such that the above equation holds, the system is stabilizable. For this set-up, we consider two cases. In the first case, called no information exchange, the encoders design functions of the form $X_{i, t}=f_{i}\left(t, x_{i, t}\right)$ as explained above. The transmission from each plant is a function only of its own data. In the second case, called information exchange case, the encoders design functions are assumed to be of the form $X_{i, t}=$ $f_{i}\left(t, x_{1, t}, x_{2, t}\right)$. In other words, the encoders can exchange the information they have access to and jointly encode the two state values.

The following are the main results of the paper. For the no information exchange case, we have the following characterization of the stability region.
Theorem 1: (No information exchange) Consider the problem formulated above for the no information exchange case. If the system (1) is stabilizable in the mean square sense through any choice of the design parameter $f_{i}(\cdot), g(\cdot)$, the following inequalities must be satisfied,

$$
\begin{aligned}
& \log \left|\lambda_{1}\right| \leq \frac{1}{2} \log \left(1+\frac{P_{1}\left(1-\rho^{2}\right)}{N}\right), \\
& \log \left|\lambda_{2}\right| \leq \frac{1}{2} \log \left(1+\frac{P_{2}\left(1-\rho^{2}\right)}{N}\right), \\
& \log \left|\lambda_{1}\right|+\log \left|\lambda_{2}\right| \leq \frac{1}{2} \log \left(1+\frac{P_{1}+P_{2}+2 \rho \sqrt{P_{1} P_{2}}}{N}\right),
\end{aligned}
$$

for some $\rho$ such that $0 \leq \rho \leq 1$.

Under the same no information exchange assumption, the system (1) is stabilizable in the mean square sense if

$$
\begin{aligned}
\log \left|\lambda_{1}\right| & <\frac{1}{2} \log \left[\left(1+\frac{P_{1}}{N}\right)^{\frac{M-1}{M}}\left(1+\frac{P_{1}\left(1-\rho^{\prime 2}\right)}{N}\right)^{\frac{1}{M}}\right], \\
\log \left|\lambda_{2}\right| & <\frac{1}{2} \log \left(1+\frac{P_{2}\left(1-\rho^{2}\right)}{N}\right)^{\frac{1}{M}},
\end{aligned}
$$

for $M \geq 1, M \in \mathbb{N}$, where the parameter $\rho^{\prime}$ is a root of the equation

$$
\begin{gathered}
\frac{\rho^{2}\left(\frac{N+P_{1}}{N}\right)^{M-1}-1}{\rho^{2}-1}=\frac{N\left(N+2|\rho| \sqrt{P_{1} P_{2}}+P_{1}+P_{2}\right)}{\left(N+P_{1}\left(1-\rho^{2}\right)\right)\left(N+P_{2}\left(1-\rho^{2}\right)\right)} . \\
\text { Proof: See Section III. }
\end{gathered}
$$

Remark 1: Ozarow [11] proved that for the Gaussian MAC channel with no information exchange between the encoders, the achievable rate region is given by

$$
\begin{aligned}
& \bigcup_{0 \leq \rho \leq 1}\left\{\left(R_{1}, R_{2}\right): 0 \leq R_{1} \leq \frac{1}{2} \log \left(1+\frac{P_{1}}{N}\left(1-\rho^{2}\right)\right),\right. \\
& 0 \leq R_{2} \leq \frac{1}{2} \log \left(1+\frac{P_{2}}{N}\left(1-\rho^{2}\right)\right), \\
& \left.0 \leq R_{1}+R_{2} \leq \frac{1}{2} \log \left(1+\frac{P_{1}+P_{2}+2 \rho \sqrt{P_{1} P_{2}}}{N}\right)\right\},
\end{aligned}
$$

If we use the data rate theorem [13] that implies that a system of the form (1) can be stabilized only if data is transmitted at a rate $R$ to the controllers such that $R>\log \lambda$, then the region (6) can be interpreted as an extension of the necessity of the data rate theorem to the MAC setting. Since there is a gap between the sufficient condition in (5) and the necessity condition in (4), the sufficiency of the data rate theorem may not extend in a similar manner.

Remark 2: The condition in [3] includes in particular the point proved to be sufficient for stability in [7]. This is the only point for which the regions described by (5) and (6) intersect and is given for the value $\rho=\rho^{*}$, where $\rho=\rho^{*}$ is the solution of the nonlinear equation

$$
\begin{array}{r}
\left(N+P_{1}\left(1-\rho^{2}\right)\right)\left(N+P_{2}\left(1-\rho^{2}\right)\right)= \\
N\left(N+2|\rho| \sqrt{P_{1} P_{2}}+P_{1}+P_{2}\right) .
\end{array}
$$

This point is obtained if we set $M=1$ in (5). 
On the other hand, for the case when information exchange is allowed between encoders, we have the following result.

Theorem 2: (Information exchange allowed) Consider the problem formulated above, for the case when information exchange is allowed between the two encoders. The system (1) is stabilizable in the mean square sense if and only if

$$
\log \left|\lambda_{1}\right|+\log \left|\lambda_{2}\right| \leq \frac{1}{2} \log \left(1+\frac{P_{1}+P_{2}+2 \sqrt{P_{1} P_{2}}}{N}\right)
$$

Proof: See Section IV.

Remark 3: This region described by (7) coincides with the region described by (4) with $\rho=1$ (see Figure 2). It is intuitive that this region is bigger in general than the region described by (4).

\section{Proof of the Necess ARY Condition OF STABILIZABILITY}

In this section, we prove Theorem 1 . We begin by proving the necessity of (4) for stabilizability of system (1). The proof is information theoretic and an extension of the proof given for point to point channels in [2].

Lemma 1: For continuous random sequence $\left\{\mathbf{x}_{t}\right\}_{t=0}^{\infty}, \mathbf{x}_{t} \in \mathbb{R}^{n}$ that converges to $\mathbf{0}$ in the mean square sense, $\lim _{t \rightarrow \infty} \mathbb{E} \mathbf{x}_{t} \mathbf{x}_{t}^{T}=\mathbf{0}$, its differential entropy $h\left(\mathbf{x}_{t}\right)$ has the property that $\lim \sup _{t \rightarrow \infty} h\left(\mathbf{x}_{t}\right)=-\infty$.

Proof: Let $\Sigma_{t}=\mathbb{E} \mathbf{x}_{t} \mathbf{x}_{t}^{T}$. Since $\lim _{t \rightarrow \infty} \Sigma_{t}=\mathbf{0}$, for any $\epsilon>0$, there exists a time $t_{0}$ such that when $t>t_{0}$, $\left|\Sigma_{t}\right|<\epsilon$. The value of differential entropy can be upper bounded by $h\left(\mathbf{x}_{t}\right)<1 / 2 \log \left(2 \pi\left|\Sigma_{t}\right|\right)<1 / 2 \log (2 \pi \epsilon)$. Since $\epsilon$ can be arbitrarily small, so $\lim _{t \rightarrow \infty} 1 / 2 \log (2 \pi \epsilon)=0$ and thus we obtain $\lim \sup _{t \rightarrow} h\left(\mathbf{x}_{t}\right)=-\infty$.

Remark 4: If $\mathbf{x}_{t}$ is a scalar, then [8, Lemma 3.2] is recovered.

Definition 2: Define the average rate of information transmitted for stabilizing the plant $i$ as $R_{i, t}$, for $i=1,2$ and every $t \geq 0$,

$$
\begin{aligned}
& R_{1, t} \triangleq \frac{1}{t} I\left(x_{1,0} ; u_{1}^{t} \mid x_{2,0}\right) \\
& R_{2, t} \triangleq \frac{1}{t} I\left(x_{2,0} ; u_{2}^{t} \mid x_{1,0}\right) .
\end{aligned}
$$

where $u_{i}^{t}$ represents $\left\{u_{i, 0}, u_{i, 1}, \ldots, u_{i, t}\right\}$.

Lemma 2:

$$
R_{i, t} \geq \log \left|\lambda_{i}\right|, \quad \text { for } i=1,2
$$

for $t$ large enough.

Proof: Consider the differential entropy of $x_{1, t}$ conditioned on $x_{2,0}$,

$$
\begin{aligned}
h\left(x_{1, t} \mid x_{2,0}\right) & =h\left(\lambda_{1}^{t} x_{1,0}+\sum_{j=0}^{t-1} \lambda_{1}^{t-j} u_{1, j} \mid x_{2,0}\right) \\
& \geq h\left(\lambda_{1}^{t} x_{1,0}+\sum_{j=0}^{t-1} \lambda_{1}^{t-j} u_{1, j} \mid \sum_{j=0}^{t-1} \lambda_{1}^{t-j} u_{1, j}, x_{2,0}\right)
\end{aligned}
$$

$$
\begin{aligned}
& =h\left(\lambda_{1}^{t} x_{1,0} \mid \sum_{j=0}^{t-1} \lambda_{1}^{t-j} u_{1, j}, x_{2,0}\right) \\
& =t \log \left(\lambda_{1}\right)+h\left(x_{1,0} \mid \sum_{j=0}^{t-1} \lambda_{1}^{t-j} u_{1, j}, x_{2,0}\right) \\
& \geq t \log \left(\lambda_{1}\right)+h\left(x_{1,0} \mid u_{1}^{t}, x_{2,0}\right)
\end{aligned}
$$

where the inequality (11) holds because conditioning does not increase the entropy; equality (12) holds because the property of differential entropy: $h(A x)=\log |A|+h(x)$; inequality (13) holds because $\sum_{j=0}^{t-1} \lambda_{1}^{t-j} u_{1, j}$ is a function of $u_{1}^{t-1}$. And $u_{1, t}$ is also added into the conditioning terms. So the entropy is reduced.

Add $h\left(x_{1,0} \mid x_{2,0}\right)$ to both sides of the inequality (13) and rearrange the terms, we have

$$
\begin{aligned}
& h\left(x_{1,0} \mid x_{2,0}\right)-h\left(x_{1,0} \mid u_{1}^{t}, x_{2,0}\right) \\
& \geq h\left(x_{1,0} \mid x_{2,0}\right)+t \log \left|\lambda_{1}\right|-h\left(x_{1, t} \mid x_{2,0}\right) .
\end{aligned}
$$

Divided by $t,(14)$ can be written as

$$
\begin{aligned}
& \frac{1}{t} I\left(x_{1,0} ; u_{1}^{t} \mid x_{2,0}\right) \geq \\
& \log \left|\lambda_{1}\right|+\frac{1}{t}\left(h\left(x_{1,0} \mid x_{2,0}\right)-h\left(x_{1, t} \mid x_{2,0}\right)\right) .
\end{aligned}
$$

Since the system (1) is stabilized in the mean square sense, which means $\lim \sup _{t \rightarrow \infty} \mathbb{E} x_{1, t}^{2}=0$. According to Lemma 1 , there exists a $T_{1}>0$, such that when $t>T_{1}$, we can find $M_{1}>0$ such that $h\left(x_{1, t} \mid x_{2,0}\right) \leq h\left(x_{1, t}\right) \leq-M_{1}$. For large enough $T_{1}$, we can have $h\left(x_{1,0} \mid x_{2,0}\right)-h\left(x_{1, t} \mid x_{2,0}\right) \geq$ $h\left(x_{1,0} \mid x_{2,0}\right)+M_{1} \geq 0$. From (14), we obtain

$$
\frac{1}{t} I\left(x_{1,0} ; u_{1}^{t} \mid x_{2,0}\right) \geq \log \left|\lambda_{1}\right|,
$$

for $t$ large enough ( when $t>T_{1}$ ). Thus, we obtain

$$
R_{1, t} \geq \log \left|\lambda_{1}\right| \text {. }
$$

By symmetry, following the similar procedure, we can see that there also exits a large number $T_{2}>0$, such that we can also obtain the inequality about system $i=2$,

$$
R_{2, t} \geq \log \left|\lambda_{2}\right| \text {. }
$$

Select $t>\max \left\{T_{1}, T_{2}\right\}$, both inequalities can be obtained at the same time. Here completes the proof.

Now, we consider the state vector $\mathbf{x}_{t}=\left[\begin{array}{ll}x_{1, t} & x_{2, t}\end{array}\right]^{T}$ of the entire system (1). Denote initial condition as $\mathbf{x}_{0}=$ $\left[\begin{array}{ll}x_{1,0} & x_{2,0}\end{array}\right]^{T}$, and input $\mathbf{u}_{t}=\left[\begin{array}{ll}u_{1, t} & u_{2, t}\end{array}\right]^{T}$.

Definition 3: Define the average rate for the state vector at $t$ as

$$
R_{12, t} \triangleq \frac{1}{t} I\left(\mathbf{x}_{0} ; \mathbf{u}^{t}\right)
$$

where $\mathbf{u}^{t}$ represents $\left\{\mathbf{u}_{0}, \mathbf{u}_{1}, \ldots, \mathbf{u}_{t}\right\}$.

Lemma 3:

$$
R_{12, t} \geq \log \left|\lambda_{1}\right|+\log \left|\lambda_{2}\right|
$$

for $t$ large enough. 
Proof: Let $A=\left[\begin{array}{cc}\lambda_{1} & 0 \\ 0 & \lambda_{2}\end{array}\right]$, consider the entropy of $\mathbf{x}_{t}$

$$
\begin{aligned}
h\left(\mathbf{x}_{t}\right) & =h\left(A^{t} \mathbf{x}_{0}+\sum_{j=0}^{t-1} A^{t-j} \mathbf{u}_{j}\right) \\
& \geq h\left(A^{t} \mathbf{x}_{0}+\sum_{j=0}^{t-1} A^{t-j} \mathbf{u}_{j} \mid \sum_{j=0}^{t-1} A^{t-j} \mathbf{u}_{j}\right) \\
& =h\left(A^{t} \mathbf{x}_{0} \mid \sum_{j=0}^{t-1} A^{t-j} \mathbf{u}_{j}\right) \\
& =t \log |A|+h\left(\mathbf{x}_{0} \mid \sum_{j=0}^{t-1} A^{t-j} \mathbf{u}_{j}\right) \\
& \geq t \log |A|+h\left(\mathbf{x}_{0} \mid \mathbf{u}^{t}\right) .
\end{aligned}
$$

Then we obtain

$$
h\left(\mathbf{x}_{0}\right)-h\left(\mathbf{x}_{0} \mid \mathbf{u}^{t}\right) \geq h\left(\mathbf{x}_{0}\right)+t \log |A|-h\left(\mathbf{x}_{t}\right),
$$

which can be written as

$$
\frac{1}{t} I\left(\mathbf{x}_{0} ; \mathbf{u}^{t}\right) \geq \log |A|+\frac{1}{t}\left(h\left(\mathbf{x}_{0}\right)-h\left(\mathbf{x}_{t}\right)\right),
$$

Suppose the system is stabilized in the mean square sense, i.e. $\lim _{t \rightarrow \infty} \mathbf{x}_{t} \mathbf{x}_{t}^{T}=\mathbf{0}$. According to Lemma 1, for $t>$ $T_{12}$, there exists an $M_{12}>0$, such that $h\left(\mathbf{x}_{1, t}\right) \leq-M_{12}$. Since $T_{12}$ can be chosen as large enough, such that $h\left(\mathbf{x}_{1,0}\right)-$ $h\left(\mathbf{x}_{1, t}\right) \geq h\left(\mathbf{x}_{1,0}\right)+M_{12} \geq 0$. From (22) and with large enough $t$, noticing that $\log |A|=\log \left|\lambda_{1}\right|+\log \left|\lambda_{2}\right|$, we obtain

$$
\frac{1}{t} I\left(\mathbf{x}_{0} ; \mathbf{u}^{t}\right) \geq \log \left|\lambda_{1}\right|+\log \left|\lambda_{2}\right| .
$$

for $t$ large enough $\left(t>T_{12}\right)$. Thus, we proved the lemma.

Lemma 4: When $t$ is large enough,

$$
\begin{aligned}
& R_{1, t} \leq \frac{1}{2} \log \left(1+\frac{P_{1}}{N}\left(1-\rho^{2}\right)\right), \\
& R_{2, t} \leq \frac{1}{2} \log \left(1+\frac{P_{2}}{N}\left(1-\rho^{2}\right)\right), \\
& R_{12, t} \leq \frac{1}{2} \log \left(1+\frac{P_{1}+P_{2}+2 \rho \sqrt{P_{1} P_{2}}}{N}\right),
\end{aligned}
$$

where $P_{1}$ and $P_{2}$ are power constraint of the channel input as mentioned in section II. $\rho=\left(\frac{1}{t} \sum_{k=1}^{t} \mu_{k}\right) / \sigma_{1, k} \sigma_{2, k}$, which is the average correlation coefficient of $X_{1, k}$ and $X_{2, k}$. $\mu_{k}=\mathbb{E} X_{1, k} X_{2, k}, \sigma_{i, k}^{2}=\mathbb{E} X_{i, k}^{2}$, for $i=1,2$.

Proof: See Appendix.

Now, we can proceed to prove the necessary condition in Theorem 1.

Proof: [Proof of the necessary condition of Theorem 1] Combine Lemma 2, 3 and Lemma 4, we can prove the necessary condition (4).

While one may conjecture that the region described by (4) is also sufficient for stabilizing system (1), proving this is not easy. In [11], the other points in (4) besides the point with $\rho=\rho^{*}$ are shown to be achievable by allowing one transmitter to send two independent messages $m_{1}^{(1)}$ and $m_{2}^{(1)}$ sharing the power $P_{1}$, and while the other transmitter sends the message $m_{2}$ with power $P_{2}$. The messages $m_{1}^{(1)}$ and $m_{2}$ are sent and decoded cooperatively using a SchalkwijkKailath-like scheme, while treating $m_{1}^{(2)}$ as a channel noise. On the other hand, the message $m_{1}^{(2)}$ is sent directly using a block code, with symbols drawn i.i.d. from a zero mean Gaussian distribution with variance $\alpha P_{1}, 0<\alpha<1$. By using successive decoding, $m_{1}^{(2)}$ can be decoded at a higher signal-to-noise ratio. Thus, the correlation coefficient $\rho$ can be varied by adjusting the power allocated to $m_{1}^{(1)}$ and $m_{1}^{(2)}$, and the other points in the region (6) can be achieved. In a control scenario, however, such a successive decoding may not be used since block codes are known to be insufficient for stabilizing an unknown plant [12].

Here, a periodic control algorithm and a time-division communication scheme are proposed to achieve more points besides the point with $\rho=\rho^{*}$ as in [7]. The entire rate region described by (4) cannot be shown to be sufficient for stability. This algorithm is used to prove the sufficient condition stated in Theorem 1.

Proof: [Proof of the Sufficient Condition in Theorem 1] Since the initial condition $x_{i, 0}, i=1,2$ is arbitrarily distributed, we can make the state Gaussian by using first two steps to do whitening process as in [7]. For notational ease, we refer the third time step as the time step the algorithm begins.

This scheme operates in a period of $M$ steps. In the $k M$-th step, the transmitters transmit an input calculated according to Ozarow's scheme, [7][11]:

$$
\begin{aligned}
& X_{1, k M}=\sqrt{\frac{P_{1}}{\alpha_{1, k M}}} x_{1, k M}, X_{2, k M}=\sqrt{\frac{P_{2}}{\alpha_{2, k M}}} x_{2, k M} \operatorname{sgn}\left(\rho_{k M}\right), \\
& Y_{k M}=\sqrt{\frac{P_{1}}{\alpha_{1, k M}}} x_{1, k M}+\sqrt{\frac{P_{2}}{\alpha_{2, k M}}} x_{2, k M} \operatorname{sgn}\left(\rho_{k M}\right)+Z_{k M},
\end{aligned}
$$

where $\rho_{k M}=\frac{\mathbb{E} x_{1, k M} x_{2, k M}}{\sqrt{\alpha_{1, k M} \alpha_{2, k M}}}$ is the correlation coefficient of the two channel inputs. The function sgn is the signum function with $\operatorname{sgn}(\rho)=1$ for $\rho$ non-negative and $\operatorname{sgn}(\rho)=-1$ for $\rho$ negative. After the decoder receives $Y_{k M}$, it computes the MMSE estimates of $\hat{x}_{i, k M}=\frac{\mathbb{E} x_{i, k M} Y_{k M}}{\mathbb{E} Y_{k M}^{2}} Y_{k M}$. We have

$$
\begin{aligned}
\mathbb{E} x_{1, k M} Y_{k M} & =\sqrt{\alpha_{1, k M}}\left(\sqrt{P_{1}}+\sqrt{P_{2}}\left|\rho_{k M}\right|\right), \\
\mathbb{E} x_{2, k M} Y_{k M} & =\sqrt{\alpha_{2, k M}}\left(\sqrt{P_{2}}+\sqrt{P_{1}}\left|\rho_{k M}\right|\right) \operatorname{sgn}\left(\rho_{k M}\right), \\
\mathbb{E} Y_{k M}^{2} & =P_{1}+P_{2}+2\left|\rho_{k M}\right| \sqrt{P_{1} P_{2}}+N
\end{aligned}
$$

where $\alpha_{i, k M}=\mathbb{E} x_{i, k M}^{2}$. For any $t \geq 0$, when the controller applies minimum variance control $u_{i, t}=-\lambda_{i} \hat{x}_{i, t}$, the state variance evolves as

$$
\begin{aligned}
\alpha_{i, t+1} & =\lambda_{i}^{2} \mathbb{E}\left(x_{i, t}-\hat{x}_{i, t}\right)^{2} \\
& =\lambda_{i}^{2} \mathbb{E}\left(x_{i, t}-\frac{\mathbb{E} x_{i, t} Y_{t}}{\mathbb{E} Y_{t}^{2}} Y_{t}\right)^{2} \\
& =\lambda_{i}^{2}\left(\mathbb{E} x_{i, t}^{2}-\frac{\left(\mathbb{E} x_{i, t} Y_{t}\right)^{2}}{\mathbb{E} Y_{t}^{2}}\right) .
\end{aligned}
$$


With $t=k M$, using (25) and (26), we obtain

$$
\begin{aligned}
& \alpha_{1, k M+1}=\alpha_{1, k M} \lambda_{1}^{2}\left(\frac{N+P_{2}\left(1-\rho_{k M}^{2}\right)}{P_{1}+P_{2}+2\left|\rho_{k M}\right| \sqrt{P_{1} P_{2}}+N}\right), \\
& \alpha_{2, k M+1}=\alpha_{2, k M} \lambda_{2}^{2}\left(\frac{N+P_{1}\left(1-\rho_{k M}^{2}\right)}{P_{1}+P_{2}+2\left|\rho_{k M}\right| \sqrt{P_{1} P_{2}}+N}\right) .
\end{aligned}
$$

At the next $M-1$ steps, $t \in[k M+1,(k+1) M-1]$, only transmitter 1 sends information about state $x_{1}$ and transmitter 2 keeps silent. We have

$$
\begin{aligned}
& X_{1, t}=\sqrt{\frac{P_{1}}{\alpha_{1, t}}} x_{1, t}, \quad X_{2, t}=0 . \\
& Y_{t}=\sqrt{\frac{P_{1}}{\alpha_{1, t}}} x_{1, t}+Z_{t} .
\end{aligned}
$$

The correlation $\mathbb{E} x_{1, t} Y_{t}$ and variance $\mathbb{E} Y_{t}^{2}$ can be obtained

$$
\begin{aligned}
\mathbb{E} x_{1, t} Y_{t} & =\sqrt{\alpha_{1, t} P_{1}}, \\
\mathbb{E} Y_{t}^{2} & =P_{1}+N .
\end{aligned}
$$

Using (26), the variances evolve as

$$
\begin{aligned}
& \alpha_{1, t+1}=\alpha_{1, t} \lambda_{1}^{2} \frac{N}{P_{1}+N}, \\
& \alpha_{2, t+1}=\alpha_{2, t} \lambda_{2}^{2} .
\end{aligned}
$$

Combining (27)(30), the state variances of the start of the next period is

$$
\begin{aligned}
& \alpha_{1,(k+1) M}=\alpha_{1, k M} \lambda_{1}^{2 M} . \\
& \left(\frac{N}{P_{1}+N}\right)^{M-1}\left(\frac{N+P_{2}\left(1-\rho_{k M}^{2}\right)}{P_{1}+P_{2}+2\left|\rho_{k M}\right| \sqrt{P_{1} P_{2}}+N}\right), \\
& \alpha_{2,(k+1) M}=\alpha_{2, k M} \lambda_{2}^{2 M}\left(\frac{N+P_{1}\left(1-\rho_{k M}^{2}\right)}{P_{1}+P_{2}+2\left|\rho_{k M}\right| \sqrt{P_{1} P_{2}}+N}\right) .
\end{aligned}
$$

Now, let us consider the evolution of $\rho_{k M}$, the correlation coefficient of $x_{1, k M}$ and $x_{2, k M}$.

$$
\begin{aligned}
& \mathbb{E} x_{1,(k+1) M} x_{2,(k+1) M} \\
& =\mathbb{E}\left[\lambda_{1}\left(x_{1,(k+1) M-1}-\hat{x}_{1,(k+1) M-1}\right) \cdot \lambda_{2} x_{2,(k+1) M-1}\right] \\
& =\lambda_{1} \lambda_{2}\left(\mathbb{E} x_{1,(k+1) M-1} x_{2,(k+1) M-1}-\mathbb{E} x_{1,(k+1) M-1} Y_{(k+1) M-1}\right. \\
& \left.\cdot \mathbb{E} x_{2,(k+1) M-1} Y_{(k+1) M-1} / \mathbb{E} Y_{(k+1) M-1}^{2}\right)
\end{aligned}
$$

Let us compute $\mathbb{E} x_{2,(k+1) M-1} Y_{(k+1) M-1}$. According to (28), we obtain

$$
\begin{aligned}
& \mathbb{E} x_{2,(k+1) M-1} Y_{(k+1) M-1} \\
& =\sqrt{\frac{P_{1}}{\alpha_{1,(k+1) M-1}} \mathbb{E} x_{1,(k+1) M-1} x_{2,(k+1) M-1}} .
\end{aligned}
$$

Substitute (29) (33) into (32), we obtain

$$
\begin{aligned}
& \mathbb{E} x_{1,(k+1) M} x_{2,(k+1) M} \\
& =\lambda_{1} \lambda_{2}\left(1-\frac{P_{1}}{P_{1}+N}\right) \mathbb{E} x_{1,(k+1) M-1} x_{2,(k+1) M-1} \\
& =\lambda_{1}^{M-1} \lambda_{2}^{M-1}\left(\frac{N}{P_{1}+N}\right)^{M-1} \mathbb{E} x_{1, k M+1} x_{2, k M+1} .
\end{aligned}
$$

The correlation $\mathbb{E} x_{1, k M+1} x_{2, k M+1}$ is shown to be [7]

$$
\begin{aligned}
& \mathbb{E} x_{1, k M+1} x_{2, k M+1}= \\
& =\lambda_{1} \lambda_{2} \sqrt{\alpha_{1, k M} \alpha_{2, k M}} \\
& \cdot\left(\frac{N \rho_{k M}-\operatorname{sgn}\left(\rho_{k M}\right) \sqrt{P_{1} P_{2}}\left(1-\rho_{k M}^{2}\right)}{P_{1}+P_{2}+2\left|\rho_{k M}\right| \sqrt{P_{1} P_{2}}+N}\right) .
\end{aligned}
$$

Then we obtain

$$
\begin{aligned}
& \rho_{(k+1) M}=\frac{\mathbb{E} x_{1,(k+1) M} x_{2,(k+1) M}}{\sqrt{\alpha_{1,(k+1) M} \alpha_{2,(k+1) M}}} \\
& =\lambda_{1}^{M} \lambda_{2}^{M}\left(\frac{N}{P_{1}+N}\right)^{M-1} \frac{\sqrt{\alpha_{1, k M} \alpha_{2, k M}}}{\sqrt{\alpha_{1,(k+1) M} \alpha_{2,(k+1) M}}} \\
& \cdot\left(\frac{N \rho_{k M}-\operatorname{sgn}\left(\rho_{k M}\right) \sqrt{P_{1} P_{2}}\left(1-\rho_{k M}^{2}\right)}{P_{1}+P_{2}+2\left|\rho_{k M}\right| \sqrt{P_{1} P_{2}}+N}\right) .
\end{aligned}
$$

With (31), the evolution of $\rho_{k M}$ can be obtained

$$
\begin{aligned}
& \rho_{(k+1) M}=\left(\frac{N}{P_{1}+N}\right)^{(M-1) / 2} . \\
& \frac{N \rho_{k M}-\operatorname{sgn}\left(\rho_{k M}\right) \sqrt{P_{1} P_{2}}\left(1-\rho_{k M}^{2}\right)}{\sqrt{\left(N+P_{1}\left(1-\rho_{k M}^{2}\right)\right)\left(N+P_{2}\left(1-\rho_{k M}^{2}\right)\right)}} .
\end{aligned}
$$

Let $\rho_{(k+1) M}=\rho_{k M}$, take square of both sides of (34) and subtract from 1 , we obtain

$$
\frac{\rho^{2}\left(\frac{N+P_{1}}{N}\right)^{M-1}-1}{\rho^{2}-1}=\frac{N\left(N+2|\rho| \sqrt{P_{1} P_{2}}+P_{1}+P_{2}\right)}{\left(N+P_{1}\left(1-\rho^{2}\right)\right)\left(N+P_{2}\left(1-\rho^{2}\right)\right)} \text {. }
$$

If $M=1$, the left hand side is $1, \rho_{k M}$ will converge to $(-1)^{k} \rho^{*}$ [11]. Setting $m>1, \rho_{k M}$ converges to $(-1)^{k} \rho^{\prime}$, with $\left|\rho^{\prime}\right|<\left|\rho^{*}\right|$. From (31), as $k$ goes large enough, $\alpha_{1, k M}$ evolves as

$$
\begin{aligned}
& \alpha_{1,(k+1) M}= \\
& \alpha_{1, k M} \lambda_{1}^{2 M}\left(\frac{N}{P_{1}+N}\right)^{M-1} \frac{N}{N+P_{1}\left(1-\rho^{2}\right)} . \\
& \frac{\rho^{\prime 2}-1}{\rho^{2}\left(\left(N+P_{1}\right) / N\right)^{M-1}-1} \\
& <\alpha_{1, k M} \lambda_{1}^{2 M}\left(\frac{N}{P_{1}+N}\right)^{M-1} \frac{N}{N+P_{1}\left(1-\rho^{\prime 2}\right)} .
\end{aligned}
$$

The sufficient condition for $\alpha_{1, k M}$ to be stable is

$$
\lambda_{1}^{2}\left(\frac{N}{P_{1}+N}\right)^{\frac{M-1}{M}}\left(\frac{N}{N+P_{1}\left(1-\rho^{2}\right)}\right)^{\frac{1}{M}}<1
$$

Similarly, using (31)(35), we obtain

$$
\frac{N+P_{1}\left(1-\rho^{\prime 2}\right)}{P_{1}+P_{2}+2\left|\rho^{\prime}\right| \sqrt{P_{1} P_{2}}+N}<\frac{N}{N+P_{2}\left(1-\rho^{\prime 2}\right)} .
$$

To ensure $\alpha_{2, k M}$ bounded, one needs

$$
\lambda_{2}^{2}\left(\frac{N}{N+P_{2}\left(1-\rho^{2}\right)}\right)^{\frac{1}{M}}<1
$$




\section{CONFIDENTIAL. Limited circulation. For review only.}

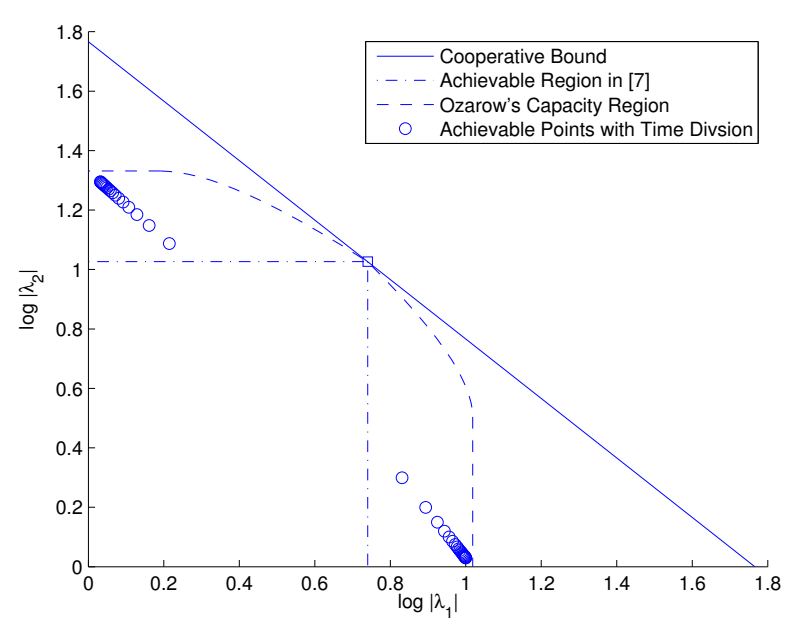

Fig. 2. Achievable points in the capacity region

Take log to both sides of (36)(37), the sufficient condition for stability with this control scheme is

$$
\begin{aligned}
& \log \left|\lambda_{1}\right|<\frac{1}{2} \log \left[\left(1+\frac{P_{1}}{N}\right)^{\frac{M-1}{M}}\left(1+\frac{P_{1}\left(1-\rho^{\prime 2}\right)}{N}\right)^{\frac{1}{M}}\right], \\
& \log \left|\lambda_{2}\right|<\frac{1}{2} \log \left(1+\frac{P_{2}\left(1-\rho^{\prime 2}\right)}{N}\right)^{\frac{1}{M}}
\end{aligned}
$$

for $M \geq 1, M \in \mathbb{Z}^{+}$.

Since $\left(1+P_{1} / N\right)>1$, there exists $M>1$ such that

$$
\left(1+\frac{P_{1}}{N}\right)^{\frac{M-1}{M}}\left(1+\frac{P_{1}\left(1-\rho^{\prime 2}\right)}{N}\right)^{\frac{1}{M}}>1+\frac{P_{1}\left(1-\rho^{* 2}\right)}{N} \text {. }
$$

Thus, with different $M$, we are able to obtain a series of points that lies outside the region with $\rho=\rho^{*}$, with the bound of $\log \left|\lambda_{1}\right|$ increased but the bound of $\log \left|\lambda_{2}\right|$ decreased. These points lie down-right to the point achieved in [7]. By symmetry, if we switch the scheme with transmitter 1 and 2, points lying upper-left can be achieved. The points achieved with this time division method are plotted in Figure 2 , with different values of $M$. We can see that these points lie between Ozarow's capacity bound (6) and the achievable region in [7].

\section{Tight Data Rate Bound With Cooperative INFORMATION STRUCTURE}

In this section, Theorem 2 is proved, under the assumption that two transmitters can exchange information.

Proof: [Proof of Theorem 2] The control and communication schemes operate in a period of $M$ steps. In every period, the time interval $[k M,(k+1) M-1]$ is divided into two segments with length $m$ and $M-m$. Two different schemes are used separately in the two segments. For any $k$-th period, during $t \in[0, m-1]$, both the transmitters send $x_{1}$. During the rest of the period $t \in[m, M-1]$, both send $x_{2}$. For $i=1,2$,

$$
X_{i, k M+t}= \begin{cases}\sqrt{\frac{P_{i}}{\alpha_{1, k M+t}}} x_{1, k M+t} & t \in[0, m-1] \\ \sqrt{\frac{P_{i}}{\alpha_{2, k M+t}}} x_{2, k M+t} & t \in[m, M-1] .\end{cases}
$$

Then the channel output is

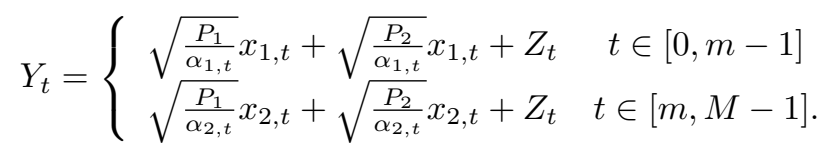

At the time step when $x_{i, t}$ is transmitted, the decoder computes the MMSE estimate $\hat{x}_{i}=\frac{\mathbb{E} x_{i, t} Y_{t}}{\mathbb{E} Y_{t}^{2}}$, with $\mathbb{E} x_{i, t} Y_{t}=$ $\left(\sqrt{P_{1}}+\sqrt{P_{2}}\right) \sqrt{\alpha_{i, t}}$ and $\mathbb{E} Y_{t}^{2}=P_{1}+P_{2}+2 \sqrt{P_{1} P_{2}}$. We also apply the minimum variance control law. Using (26), the variance of $x_{i, t+1}$ is

$$
\alpha_{i, t+1}=\lambda_{i}^{2} \alpha_{i, t}\left(\frac{N}{P_{1}+P_{2}+2 \sqrt{P_{1} P_{2}}+N}\right) .
$$

When the other state is transmitted, $x_{i, t}$ goes open loop. In next period, the state variances are

$$
\begin{aligned}
& \alpha_{1,(k+1) M}=\alpha_{1, k M} \lambda_{1}^{2 M}\left(\frac{N}{P_{1}+P_{2}+2 \sqrt{P_{1} P_{2}}+N}\right)^{m}, \\
& \alpha_{2,(k+1) M}=\alpha_{2, k M} \lambda_{2}^{2 M}\left(\frac{N}{P_{1}+P_{2}+2 \sqrt{P_{1} P_{2}}+N}\right)^{M-m} .
\end{aligned}
$$

Under this periodic control law, the sufficient condition of the mean square stability is

$$
\begin{aligned}
& \lambda_{1}^{2 M}\left(\frac{N}{P_{1}+P_{2}+2 \sqrt{P_{1} P_{2}}+N}\right)^{m}<1, \\
& \lambda_{2}^{2 M}\left(\frac{N}{P_{1}+P_{2}+2 \sqrt{P_{1} P_{2}}+N}\right)^{M-m}<1 .
\end{aligned}
$$

This leads to the condition below

$$
\begin{array}{r}
\log \left|\lambda_{1}\right|<\frac{1}{2} \frac{m}{M} \log \left(1+\frac{P_{1}+P_{2}+2 \sqrt{P_{1} P_{2}}}{N}\right), \\
\log \left|\lambda_{2}\right|<\frac{1}{2} \frac{M-m}{M} \log \left(1+\frac{P_{1}+P_{2}+2 \sqrt{P_{1} P_{2}}}{N}\right) .
\end{array}
$$

Adjusting the value $m$ and $M$, all of the points on the cooperative bound line can be achieved. Therefore, we proved the condition (4) in Theorem 2 is sufficient .

Now, we prove that the condition (4) is also necessary. Notice that in dealing with sum rate bound of $\log \left|\lambda_{1}\right|+\log \left|\lambda_{2}\right|$ in the necessity proof of Theorem 1 , there is no dependence on the information exchange assumption, i.e. there is no requirement on the exact form of encoding function $f_{i}$ and feedback control law. So Lemma 3 and the third inequality in (24) still hold in this case. Combining them, we obtain $\log \left|\lambda_{1}\right|+\log \left|\lambda_{2}\right| \leq \frac{1}{2} \log \left(1+\frac{P_{1}+P_{2}+2 \rho \sqrt{P_{1} P_{2}}}{N}\right)$, which is the third inequality in (4).

Since the maximum value of $\rho$ is 1 , then we have

$$
\log \left|\lambda_{1}\right|+\log \left|\lambda_{2}\right|<\left(1+\frac{P_{1}+P_{2}+2 \sqrt{P_{1} P_{2}}}{N}\right),
$$

which completes the proof. 


\section{CONCLUSIONS}

In this paper, we consider the problem of stabilizing two linear scalar plants over a Gaussian multiple access channel. A necessary condition is obtained, with a region for logarithms of eigenvalues that coincides with the capacity region Gaussian of Gaussian multiple access feedback channel. For the sufficiency, we achieve new points in the capacity region by using time switching algorithms. Furthermore, under the assumption that two transmitters can exchange information with each other, we obtain a tight data rate bound for stabilizing the two plants.

\section{APPENDIX}

In this appendix, we prove Lemma 4. Most of the derivation is borrowed from the necessity proof in [11], with a few adjustments for our problem. First, we prove the following lemma needed in proving Lemma 4.

Lemma 5: The following inequalities hold, for $t$ large enough.

$$
\begin{aligned}
R_{1, t} & \leq \frac{1}{t} I\left(x_{1,0} ; Y^{t} \mid x_{2,0}\right), \\
R_{2, t} & \leq \frac{1}{t} I\left(x_{2,0} ; Y^{t} \mid x_{1,0}\right), \\
R_{12, t} & \leq \frac{1}{t} I\left(x_{1,0}, x_{2,0} ; Y^{t}\right) .
\end{aligned}
$$

Proof: Since $u_{i}^{t}$ is a function of $Y^{t}$, for all $t$,

$$
\begin{aligned}
I\left(x_{1,0} ; u_{1}^{t} \mid x_{2,0}\right) & \leq I\left(x_{1,0} ; Y^{t} \mid x_{2,0}\right), \\
I\left(x_{2,0} ; u_{2}^{t} \mid x_{1,0}\right) & \leq I\left(x_{2,0} ; Y^{t} \mid x_{1,0}\right), \\
I\left(\mathbf{x}_{0} ; \mathbf{u}^{t}\right) & \leq I\left(x_{1,0}, x_{2,0} ; Y^{t}\right) .
\end{aligned}
$$

Divide both sides of all inequalities by $t$ and let $t$ be large enough, $t>\max \left\{T_{1}, T_{2}, T_{12}\right\}$. With Lemma 2 and Lemma 3 , the three inequalities are proved.

With the lemma above, we proceed to prove Lemma 4.

Proof: [Proof of Lemma 4]

Consider the differential entropy of $Y^{t}$

$$
\begin{aligned}
h\left(Y^{t}\right) & =\sum_{k=1}^{t} h\left(Y_{k} \mid Y^{k-1}\right) \\
& \leq \sum_{k=1}^{t} h\left(Y_{k}\right) .
\end{aligned}
$$

The inequality above holds because of the chain rule. For plant $i$,

$$
\begin{aligned}
h\left(Y^{t} \mid x_{i, 0}\right) & =\sum_{k=1}^{t} h\left(Y_{k} \mid Y^{k-1}, x_{i, 0}\right) \\
& =\sum_{k=1}^{t} h\left(Y_{k} \mid X_{i, k}, Y^{k-1}, x_{i, 0}\right) \\
& \leq \sum_{k=1}^{t} h\left(Y_{k} \mid X_{i, k}\right) .
\end{aligned}
$$

The equality (40) holds because $X_{i, k}$ is a function of $Y^{k-1}$ and $x_{i, 0}$. Also we have

$$
\begin{aligned}
h\left(Y^{t} \mid x_{1,0}, x_{2,0}\right) & =\sum_{k=1}^{t} h\left(Y_{k} \mid Y^{k-1}, x_{1,0}, x_{2,0}\right) \\
& =\sum_{k=1}^{t} h\left(Y_{k} \mid X_{1, k}, X_{2, k}, Y^{k-1}, x_{1,0}, x_{2,0}\right) \\
& =\sum_{k=1}^{t} h\left(Y_{k} \mid X_{1, k}, X_{2, k}\right) .
\end{aligned}
$$

The equality (41) $X_{1, k}, X_{2, k}$ are functions of $Y^{k-1}, x_{1,0}$ and $x_{2,0}$. The last equality (42) holds because the nature of the memoryless channel. Since

$$
\begin{aligned}
I\left(x_{1,0} ; Y^{t} \mid x_{2,0}\right) & =h\left(Y^{t} \mid x_{2,0}\right)-h\left(Y^{t} \mid x_{1,0}, x_{2,0}\right) \\
& \leq \sum_{k=1}^{t} h\left(Y_{k} \mid X_{2, k}\right)-h\left(Y_{k} \mid X_{1, k}, X_{2, k}\right) \\
& =\sum_{k=1}^{t} I\left(Y_{k} ; X_{1, k} \mid X_{2, k}\right) \\
I\left(x_{1,0}, x_{2,0} ; Y^{t}\right) & =h\left(Y^{t}\right)-h\left(Y^{t} \mid x_{1,0}, x_{2,0}\right) \\
& \leq \sum_{k=1}^{t} h\left(Y_{k}\right)-h\left(Y_{k} \mid X_{1, k}, X_{2, k}\right) \\
& =\sum_{k=1}^{t} I\left(Y_{k} ; X_{1, k}, X_{2, k}\right)
\end{aligned}
$$

In summary, we show that the mutual informations $I\left(x_{1,0} ; Y^{t} \mid x_{2,0}\right) I\left(x_{2,0} ; Y^{t} \mid x_{1,0}\right)$ and $I\left(x_{1,0}, x_{2,0} ; Y^{t}\right)$ can be bounded by

$$
\begin{aligned}
& I\left(x_{1,0} ; Y^{t} \mid x_{2,0}\right) \leq \sum_{k=1}^{t} I\left(Y_{k} ; X_{1, k} \mid X_{2, k}\right), \\
& I\left(x_{2,0} ; Y^{t} \mid x_{1,0}\right) \leq \sum_{k=1}^{t} I\left(Y_{k} ; X_{2, k} \mid X_{1, k}\right), \\
& I\left(Y_{k} ; X_{1, k}, X_{2, k}\right) \leq \sum_{k=1}^{t} I\left(Y_{k} ; X_{1, k}, X_{2, k}\right) .
\end{aligned}
$$

With Lemma 5, for $t$ large enough, we have

$$
\begin{aligned}
& R_{1, t} \leq \frac{1}{t}\left(\sum_{k=1}^{t} I\left(X_{1, k} ; Y_{k} \mid X_{2, k}\right)\right), \\
& R_{2, t} \leq \frac{1}{t}\left(\sum_{k=1}^{t} I\left(X_{2, k} ; Y_{k} \mid X_{1, k}\right)\right), \\
& R_{12, t} \leq \frac{1}{t}\left(\sum_{k=1}^{t} I\left(X_{2, k}, X_{1, k} ; Y_{k}\right)\right) .
\end{aligned}
$$

Next, we will show the terms of the right hand side can be upper bounded by terms related to Gaussian channels 
$P_{1}, P_{2}, N$. Notice that

$$
\begin{aligned}
& I\left(X_{1, k} ; Y_{k} \mid X_{2, k}\right)=h\left(Y_{k} \mid X_{2, k}\right)-h\left(Y_{k} \mid X_{1, k}, X_{2, k}\right) \\
& I\left(X_{1, k}, X_{2, k} ; Y_{k}\right)=h\left(Y_{k}\right)-h\left(Y_{k} \mid X_{1, k}, X_{2, k}\right)
\end{aligned}
$$

For any $k$, since $Y_{k}=X_{1, k}+X_{2, k}+Z_{k}$, we have

$$
h\left(Y_{k} \mid X_{1, k}, X_{2, k}\right)=h\left(Z_{k}\right)=\frac{1}{2} \log 2 \pi e N .
$$

Let $\sigma_{i, k}^{2}=\operatorname{var}\left(X_{i}\right)$, and denote the covariance of $X_{1, k}$ and $X_{2, k}$ as $\mu_{k}$, then

$$
\begin{aligned}
h\left(Y_{k}\right) & =h\left(X_{1, k}+X_{2, k}+Z_{k}\right) \\
& \leq \frac{1}{2} \log 2 \pi e \operatorname{var}\left(X_{1, k},+X_{2, k}+Z_{k}\right) \\
& =\frac{1}{2} \log 2 \pi e \operatorname{var}\left(\sigma_{1, k}^{2}+\sigma_{2, k}^{2}+N+2 \mu_{k}\right) .
\end{aligned}
$$

Now assume that $X_{1, k}=x_{1}$. The conditional variance of $X_{2, k}$ given $X_{1, k}$ is no greater than the variance of $X_{2, k}$ around the linear estimate $\hat{x}_{2}=\left(\mu / \sigma_{1}^{2}\right) x_{1}$. Then we have

$$
\begin{aligned}
\operatorname{var}\left(X_{2, k} \mid X_{1, k}\right) & :=\mathbb{E}_{x_{1}} \operatorname{var}\left(X_{2, k} \mid X_{1, k}=x_{1}\right) \\
& \leq \mathbb{E}_{x_{1}} \mathbb{E}_{x_{2} \mid x_{1}}\left(X_{2, k}-\frac{\mu_{k} x_{1}}{\sigma_{1, k}^{2}}\right)^{2} \\
& =\sigma_{2, k}^{2}\left(1-\frac{\mu_{k}^{2}}{\sigma_{1, k}^{2} \sigma_{2, k}^{2}}\right)
\end{aligned}
$$

Therefore,

$$
\begin{aligned}
h\left(Y_{k} \mid X_{1, k}\right) & =\mathbb{E}_{x_{1}} h\left(Y_{k} \mid X_{1, k}=x_{1}\right) \\
& \leq \mathbb{E}_{x_{1}} \frac{1}{2} \log 2 \pi e \operatorname{var}\left(X_{1, k}+X_{2, k}+Z_{k} \mid X_{1, k}=x_{1}\right) \\
& \leq \mathbb{E}_{x_{1}} \frac{1}{2} \log 2 \pi e\left[\operatorname{var}\left(X_{2, k} \mid X_{1, k}=x_{1}\right)+N\right]
\end{aligned}
$$

where inequality (55) holds because $Z_{k}$ is independent of $X_{1, k}$. By Jensen's inequality,

$$
\begin{aligned}
h\left(Y_{k} \mid X_{1, k}\right) & \leq \frac{1}{2} \log 2 \pi e\left[\mathbb{E}_{x_{1}} \operatorname{var}\left(X_{2, k} \mid X_{1, k}=x_{1}\right)+N\right] \\
& \leq \frac{1}{2} \log 2 \pi e\left(\sigma_{2, k}^{2}\left(1-\frac{\mu_{k}^{2}}{\sigma_{1, k}^{2} \sigma_{2, k}^{2}}\right)+N\right) .
\end{aligned}
$$

Also we can obtain the similar bound for $h\left(Y_{k} \mid X_{2, k}\right)$ by switching $\sigma_{1, k}^{2}$ and $\sigma_{2, k}^{2}$. Then we have

$$
\begin{aligned}
& R_{1, t} \leq \frac{1}{t} \sum_{k=1}^{t} \frac{1}{2} \log \left[\frac{\sigma_{1, k}^{2}\left(1-\frac{\mu_{k}^{2}}{\sigma_{1, k}^{2} \sigma_{2, k}^{2}}\right)+N}{N}\right], \\
& R_{2, t} \leq \frac{1}{t} \sum_{k=1}^{t} \frac{1}{2} \log \left[\frac{\sigma_{2, k}^{2}\left(1-\frac{\mu_{k}^{2}}{\sigma_{1, k}^{2} \sigma_{2, k}^{2}}\right)+N}{N}\right], \\
& R_{12, t} \leq \frac{1}{t} \sum_{k=1}^{t} \frac{1}{2} \log \left[\frac{\sigma_{1, k}^{2}+\sigma_{2, k}^{2}+2 \mu_{k}+N}{N}\right] .
\end{aligned}
$$

Since the terms in the right side is concave in $\sigma_{1, k}^{2}, \sigma_{2, k}^{2}$ and $\mu_{k}$, so Jensen's inequality can be used. Define

$$
\rho=\left(\frac{1}{t} \sum_{k=1}^{t} \mu_{k}\right) / \sigma_{1, k} \sigma_{2, k} .
$$

It is easy to see that $0 \leq \rho \leq 1$. Thus, as $t$ is large enough, we obtain

$$
\begin{array}{r}
R_{1, t} \leq \frac{1}{2} \log \left(1+\frac{P_{1}}{N}\left(1-\rho^{2}\right)\right), \\
R_{2, t} \leq \frac{1}{2} \log \left(1+\frac{P_{2}}{N}\left(1-\rho^{2}\right)\right), \\
R_{12, t} \leq \frac{1}{2} \log \left(1+\frac{P_{1}+P_{2}+2 \rho \sqrt{P_{1} P_{2}}}{N}\right) .
\end{array}
$$

\section{REFERENCES}

[1] J. Hespanha, P. Naghshtabrizi, and Y. Xu, "A survey of recent results in networked control systems," Proceedings of the IEEE, vol. 95, no. 1, pp. $138-162$, jan. 2007.

[2] J. Braslavsky, R. Middleton, and J. Freudenberg, "Feedback stabilization over signal-to-noise ratio constrained channels," Automatic Control, IEEE Transactions on, vol. 52, no. 8, pp. 1391 -1403, aug. 2007.

[3] N. Elia, "When bode meets shannon: control-oriented feedback communication schemes," Automatic Control, IEEE Transactions on, vol. 49, no. 9, pp. 1477 - 1488, sept. 2004.

[4] S. Tatikonda and S. Mitter, "Control over noisy channels," Automatic Control, IEEE Transactions on, vol. 49, no. 7, pp. 1196 - 1201, july 2004.

[5] U. Kumar, V. Gupta, and J. Laneman, "On stability across a gaussian product channel," in Decision and Control and European Control Conference (CDC-ECC), 2011 50th IEEE Conference on, dec. 2011, pp. $3142-3147$.

[6] - "Sufficient conditions for stabilizability over gaussian relay and cascade channels," in Decision and Control (CDC), 2010 49th IEEE Conference on, dec. 2010, pp. $4765-4770$.

[7] A. Zaidi, T. Oechtering, and M. Skoglund, "Sufficient conditions for closed-loop control over multiple-access and broadcast channels," in Decision and Control (CDC), 2010 49th IEEE Conference on, dec. 2010, pp. $4771-4776$

[8] S. Yuksel and T. Basar, "Optimal signaling policies for decentralized multicontroller stabilizability over communication channels," Automatic Control, IEEE Transactions on, vol. 52, no. 10, pp. 1969 -1974, oct. 2007.

[9] G. Nair, R. Evans, and P. Caines, "Stabilising decentralised linear systems under data rate constraints," in Decision and Control, 2004 CDC. 43rd IEEE Conference on, vol. 4, dec. 2004, pp. 3992 - 3997 Vol.4.

[10] J. Liu and V. Gupta, "Decentralized control over analog erasure channels,", in Decision and Control, 2012. CDC. 43rd IEEE Conference on, accepted, dec. 2012.

[11] L. Ozarow, "The capacity of the white gaussian multiple access channel with feedback," Information Theory, IEEE Transactions on, vol. 30, no. 4, pp. 623 - 629, jul 1984.

[12] A. Sahai and S. Mitter, "The necessity and sufficiency of anytime capacity for stabilization of a linear system over a noisy communication link, part i: Scalar systems," Information Theory, IEEE Transactions on, vol. 52, no. 8, pp. 3369-3395, aug. 2006.

[13] S. Tatikonda and S. Mitter, "Control under communication constraints," Automatic Control, IEEE Transactions on, vol. 49, no. 7, pp. 1056 - 1068, july 2004 . 